\title{
Enhancing and Developing Multi Block Proxy Re-encryption Methodology in Network Security
}

\author{
R. Durga, B. Vinothini
}

\begin{abstract}
Windows Azure Storage (WAS) is a scattered accumulating structure that empowers clients to store clearly boundless tends of information for any term of time. Gigantic safety is not given internet to content prospering. Internet storage device separates record into social events and contemplated security. Those encoded gatherings placed on emulate storage device assistance. The mixed contents changed over converted formats included esteem byte execution getting to the essential content. The internet device makes correspondence included mixed contents showed up unmistakably in group with the etching outfitted with the access to confirm the content ordinariness. The code is executed for the back-up of the data. The encryption arrangement of the contents done by the content owner before it accomplishes internet storage. This ensures veritable twofold clock encryption. In the paper we deal with the existing system we deals with. Server used for storing data. Single Content are uploaded into the different size in different server. That is inability to any information in a server influence the entire framework. Along these lines all content setback from the server. In the wake of utilizing different server this issue could be stayed away from. The encryption plots in the current framework are not compelling in light of the fact that just couples of activities are upheld over scrambled information. In the event that at all security exists, the outsider evaluator ought to be permitted to get to the whole information bundles for confirmation. Internet encounters a ceaseless disillusionment escape contents ground-breaking support execution present structure. This framework, Internet storage will part of document converts same size, thought about security. These relating encoded groups placed in various Internet storage, its passwords are dissipated in various main devices. That encoded information are changed over formats included consistency execution bind access getting to the primary content by utilizing AES, MD5 ALGORITHM if the considerable customer getting to the content cloud will recoup the contents reversible way.
\end{abstract}

Keywords--- Ensure Codes, Content Splitting, Encryption, AES, MD5.

\section{INDRODUCTION}

Eradication codes [1] are generally supported as a reasonable way to ensure the information dependability of capacity frameworks (e.g., plate exhibit [2], [3], [4], [5], [6], capacity [12], [13], [14]). They ensure encoded principal content squares to make new equality squares, with the goal that a subset of the squares is adequate to recoup every single unique datum. Deletion codes are generally received away frameworks to spare the storage room and vitality cost [12], [13], [14]. In any case, such productive codes experience the ill effects of high circle I/O overhead amid information refresh [14], [15], [16], [17]. Therefore, long

\footnotetext{
Manuscript received September 16, 2019.

Dr.R. Durga, Assistant Professor, Department of Computer Science, Vels University, Chennai. T.N, India. (e-mail: durdurga@gmail.com)

B. Vinothini, Research Scholar, Department of Computer Science, Vels University, Chennai. T.N, India. (e-mail: vinothinib92@gmail.com)
} [7], [8], circled limit structures [9], [10], [11], and circulated

information refresh inactivity is difficult to be merged. This speaks to a gigantic hindrance to its broad gathering on internet applications (e.g., casual connections) lethargy, an essential importance.

Content revives were overwhelming tasks. True estimations demonstrate that up to 90 percent of the compose solicitations to capacity frameworks may incorporate invigorate exercises [14]. Decreasing the input/output for invigorate exercises is from this time forward a fundamental stress towards applying cancellation codes in online applications. So as to accomplish the confirmations of cloud information honesty and accessibility and implement the nature of distributed storage administration, effective strategies that empower on-request information rightness check for cloud clients must be structured. Be that as it might, the way that customers never again have physical responsibility for in the cloud blocks the quick appointment of standard cryptographic natives with the end goal of information trustworthiness insurance. Thus, the confirmation of distributed storage rightness must be led without unequivocal information of the entire information documents; meanwhile, distributed capacity isn't just an outcast content stockroom. The content set away in the cloud may not exclusively be gotten to yet additionally be every now and again refreshed by the clients, including inclusion, cancellation, alteration, adding, and so on. it is similarly essential to help the blend of this dynamic component into the appropriated stockpiling exactness assertion, which makes the structure design stunningly much progressively troublesome. Last at any rate not the least; the sending of circled preparing is filled by server farms running in a synchronous, formed, and flowed way.

It is continuously focal concentrations separate customers for save content monotonously over different manual device so as to diminish contents decency includes openness perils. Along these lines, dispersed traditions for limit rightness assertion are essentialness for getting fiery protects disseminated stockpiling devices. Nevertheless, similar basic region stays totally researched composition. A normal technique for appearing in appropriated gathering structures has been to go over the information, save different duplicates information explicit devices spills transversely over various disappointment spaces. While the straightforwardness of the replication system is attracting, the fast progression in the extent of information holding up be verified has made verifying different duplicates of the information an over the top strategy.

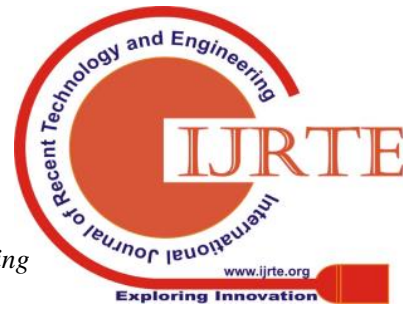




\section{ENHANCING AND DEVELOPING MULTI BLOCK PROXY RE-ENCRYPTION METHODOLOGY IN NETWORK SECURITY}

The volume of information holding up be verified is making at a quick rate, defeating the profitability rate relating to Moore's law for cutoff gadgets. All things considered, paying little respect to the technique with decrease in the expense of point of confinement contraptions, replication is too excessive a reaction for gigantic scale putting away frameworks. In the present structure, Content are moved into the differing servers with different sizes gigantic encryption given in the Internet devices to content prosperity. Multiple servers utilized for putting away information. So disappointment happened. That is inability to any information in a solitary server influence the entire framework. Hence all information the encryption conspires in the current framework are not viable in light of the fact that just couple of tasks are upheld over encoded information. On the off chance that at all security exists, the outsider evaluator ought to be permitted to get to the whole information bundles for check. Cloud experiences a perpetual no compelling reinforcement process in the current framework.

The encryption conspires in the current framework are not powerful in light of the fact that just couple of activities are upheld over scrambled information. On the off chance that at all security exists, the outsider evaluator ought to be permitted to get to the whole information bundles for confirmation. Cloud experiences a lasting no successful reinforcement process in the current framework. The encryption plots in the current framework are not powerful in light of the fact that just couples of activities are bolstered over encoded information. On the off chance that at all security exists, the outsider evaluator ought to be permitted to get to the whole information parcels for confirmation. Cloud experiences a lasting no viable reinforcement process in the current framework.

Under customary eradication codes, for example, RS codes, repetition is presented in the accompanying way: A document to be put away is isolated into equivalent estimated values, arrangement values; $r$ equality units (scientific elements of the $\mathrm{k}$ unique units) are registered. The arrangement of these $(k+r)$ units establishes a stripe. The information and equality units having a place with a stripe are set on various servers, commonly browsed diverse disappointment areas.

The consistency units have the units in a stripe such to recoup the fundamental information. As necessities be, disappointment of suffered with no content pain. In the proposed structure, Cloud server will part the record into social occasions and thought about encryption. The relating encoded packs are kept in various Cloud servers and their keys are appropriated in various key server. This blended information are changed over into bytes and included equity bit process by the information proprietor to control TPA by getting to the fundamental information. The Cloud server makes the token number from the equity included encoded information and showed up contrastingly in relationship with the scratching gave the TPA to request the Content Integrity. Likewise execute Erasure Code for the help of the information. The encryption game-plan of the information by the information proprietor done before it achieves the Cloud server. This guarantees genuine twofold time security. In the AES ALGORITHM used to encode the information move near information in various servers with various sizes and if the obliteration code utilized is a Maximum-Distance-Separable (MDS) code [100], the purpose of imprisonment structure will be impeccable in using the extra space for offering change in accordance with inside disillusionment. In particular, under a $(\mathrm{k}, \mathrm{r})$ MDS code, each inside point stores a ( $1 \mathrm{k}$ )the bit of the data, and has the property that the entire content can be decoded from any $k$ out of the $n(=k+r)$ focus focuses.

Consequently, such a code can continue on through the disappointment of any $r$ of the $n$ center points with no information misfortune.

\section{RELATED WORK \& RESULTS}

\section{Recovering Codes}

Key duty [1], Demakis showed recuperating scripts show, which streamlines proportion midst of fix exercises. In the makers give a framework stream (cutest) based lower bound for the proportion of content download in the midst of what is known as a viable fix. Under helpful fix, the fixed center point is simply for all intents and purposes indistinguishable to the failed center point. In the makers exhibited the speculative nearness of codes meeting the cutest set out toward the viable fix setting. We will consider a continuously stringent need named unequivocal fix, wherein the replicated center is undefined center point. To fix content transmission prompts an additional middle trade speed utilized for fix activities, and it's known as farthest point content trade limit tradeoff. Two essential spotlights off are its end focuses named the Minimum-StorageRegenerating (MSR) and the Minimum-Bandwidth Regenerating (MBR) focuses. MSR scripts are MDS, and all things considered cutoff the extent of additional room ate up. For this inconsequential extent of additional room, MSR codes moreover limit the extent of information downloaded amidst fix.

\section{Improving reconstruction efficiency}

Combined scripts gotten phenomenal thought composing as a result of their wide use in circle show structures, for example, EVEN-ODD and RDP codes. The EVEN-ODD and RDP codes have been refreshed for amusement independently. A progressing work presents a revamping structure for standard Reed-Solomon codes for decreasing the proportion of content trade in the midst of multiplication segments instead of constrained created. This work upgrades only the proportion of content proportion of content study in the midst of revamping.

\section{Saving Devices}

Plate displays had used cancellation -gainful adjustment to non-basic disappointment as Redundant Array of Inexpensive Disks (RAID) systems .The benefits of annihilation offering adjustment to inside disappointment in dispersed amassing structures has in like manner been all around considered and erasure codes have been used in various settings, for instance, orchestrate associated limit

Published By: 
structures, shared limit structures, etc. Starting late, destruction coding is being growing sent in datacenter-scale dispersed limit structures to achieve adjustment to non-basic disappointment while constraining accumulating necessities.

\section{Algorithm technique}

AES relies on a game-plan sort out that was appropriate in equipment and programming. Rather than that is harbinger DES, AES can't deal with. AES is an arrangement of which have a fixed square size of 128 bits, and a key size of 128,192 , or 256 bits. Then again, is settled with square and key sizes that may be any unique of 32 5bits, with something like 128 and a point of containment of 256 bits.AES wears out a $4 \times 4$ part veritable intrigue show of bytes, named the state. Most AES calculations are done in a particular constrained field. For instance, if there are 16 bytes, these bytes are tended to as this two-dimensional pack.

$$
\left[\begin{array}{llll}
b_{0} & b_{4} & b_{8} & b_{12} \\
b_{1} & b_{5} & b_{9} & b_{13} \\
b_{2} & b_{6} & b_{10} & b_{14} \\
b_{3} & b_{7} & b_{11} & b_{15}
\end{array}\right]
$$

The password length utilized AES picture demonstrates measure of advancement changes which convert information, converts field, and known as figure content.

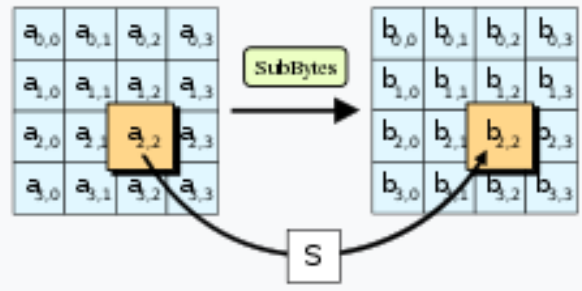

It is used to encrypt the content in different servers with different size.

\section{MD5 Algorithm}

Pulverization programming was system for content security parts, augmented and secured monotonous contents and set away over great deal zones limit. Target engage content breezes up undermined finally in the plate hiding away system to be recreated by using information about content that is confirmed elsewhere in the group. Destruction codes are dependably utilized rather than standard RAID in light of capability to diminish the time and overhead required to rehash data. The weakness of destruction coding, is that it might inspired, and that can change over into extended torpidity. Specifically, under a (k, r) MDS code, each middle point stores a $(1 \mathrm{k})$ th part of the information, and has the property that the whole information can, be decoded from any $\mathrm{k}$ out of the $\mathrm{n}(=\mathrm{k}+\mathrm{r})$ center points As such, such a code can hold up under the slip-up of any $\mathrm{r}$ of the $\mathrm{n}$ center, points with no content setback. A lone, stripe of a $(\mathrm{k}=4, \mathrm{r}=2)$ MDS code is depicted in Figure 2.1, where $\{a 1, a 2, a 3, a 4\}$ are the restricted field parts identifying with the content encrypted. See that each center point piece of the total data, and all of the content would recover content set away.

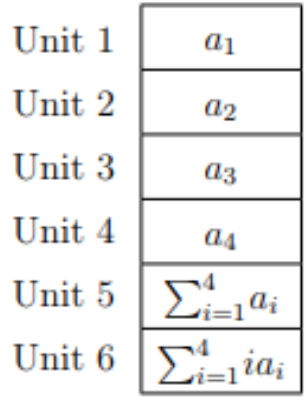

The redundancy or information put away, for example:

Storage overhead or redundancy $=\frac{n}{k}$.

MSD Program code

$$
\text { Rate }=\frac{k}{n}
$$

\section{MD5 Hashing}

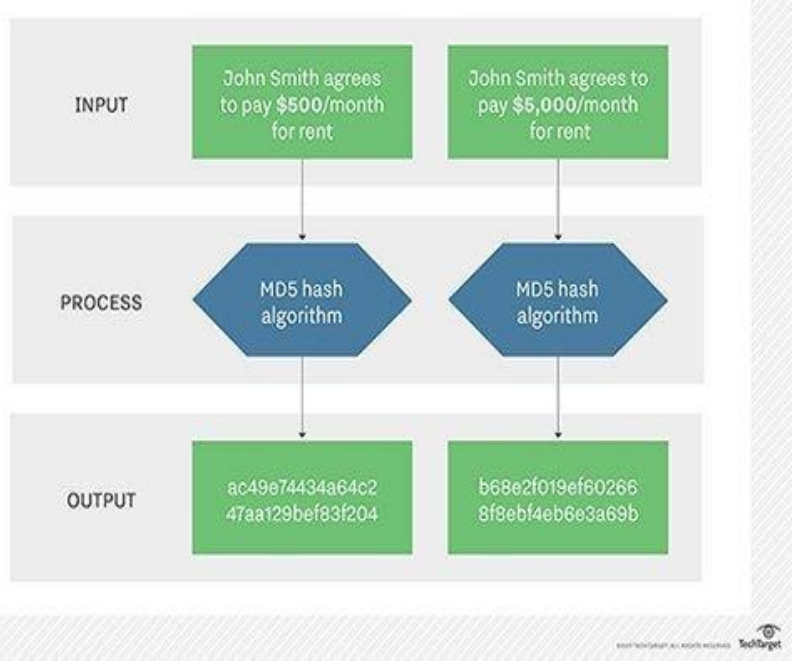

\section{Significance of Design}

The incessant transitory and perpetual disappointments that happen in server farms render numerous pieces of the information inaccessible every once in a while. Review that we are concentrating on a solitary stripe, and for this situation, inaccessibility relates to inaccessibility of at least one hubs in the stripe. So as to keep up the focused on dimension of unwavering quality and accessibility, a missing hub should be supplanted by another hub by reproducing the information that was put away in it, with the assistance of the rest of the hubs. It considers activity reproduction task. We will likewise utilize recuperation fix tasks conversely allude reproduction activities. huge frameworks, reproduction tasks are kept running as foundation occupations. In the existing system we deals with content are uploaded in different servers with different size. No enormous security gave in the Cloud server to information wellbeing. Just one device securing content. Hence one value disillusionment happened. A lone server impacts the entire framework.

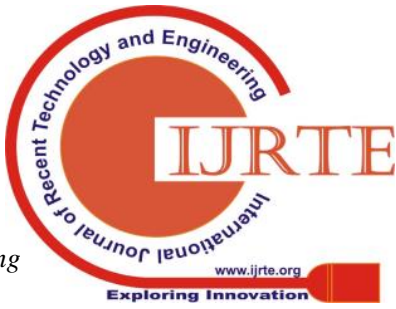




\section{ENHANCING AND DEVELOPING MULTI BLOCK PROXY RE-ENCRYPTION METHODOLOGY IN NETWORK SECURITY}

In this way all information misfortune from the server. Subsequent to utilizing various servers this issue could be maintained a strategic distance from. The encryption conspires in the current framework are not successful on the grounds that just couple of tasks are bolstered over scrambled information. In the event that at all security exists, the outsider inspector ought to be permitted to get to the whole information parcels for confirmation. Cloud experiences a unchanging disillusionment and lost all of content is no viable reinforcement process in the current framework.

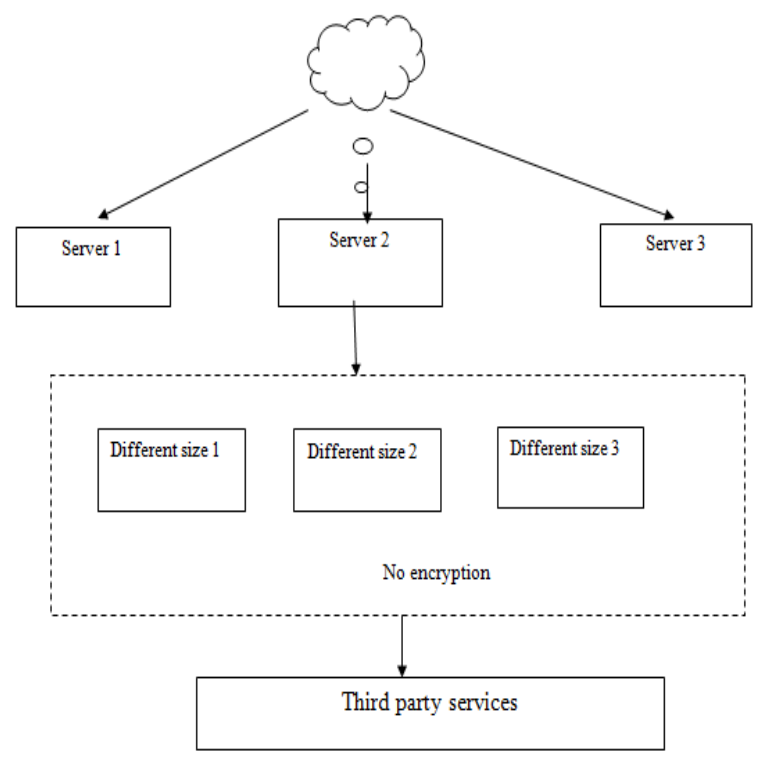

\section{Implementation of Design}

Upon segment, delivered some estimations, discernments content bunch progress saves a few pet bytes of content over several thousand machines, concentrating on the inaccessibility estimations furthermore, the effect of utilizing destruction lines (explicitly) framework establishment server ranch. Estimations, watched center of than 50 machine inaccessibility occasions for reliably. Those separation estimations support past results server farms concerning inaccessibility standard as opposed to the remarkable case.

The examination estimations additionally uncovered demolition secured information, one dissatisfactions a wide edges the larger situation. Affected by this, we will concentrate on redesigning for re-trying of single disappointment going with territories. We besides seen that the tremendous extent of download performed by the RSencoded information amidst age of missing squares gobbles up an all around high extent of system trade speed and unequivocally, puts extra burden, on the legitimately oversubscribed top-of-rack switches. This gives a credible inspiration to our work on patching up gain full, devastation codes for appropriated putting away displayed. In the present structure we regulates, information are moved in various servers with various size. No gigantic security, gave in the Cloud server to content achievement. Basically single server used for confirming data. Frustration occurred. That is powerlessness content in, a specific server influence the whole system. As such all content event from the server. In the wake of using different servers this issue could, be avoided The encryption brainstorms in the present structure are not convincing, in light of the manner in which that simply couple of assignments are maintained over mixed data. If at all security exists, the pariah commentator ought to be permitted, to get to the whole information, packs for confirmation. Cloud experiences, an endless the majority of its information there is no persuading help process in the present structure.

\section{Advantages}

One way to deal with oversee give content imperativeness, is to mimic a message with the veritable focus on that each most distant point. A decentralized, destruction code is sensible for use in a left on putting away behind system. A re-encryption plot and harden it with a safe decentralized code to shape a safe circled stockpiling system is proposed. The encryption, plot supports encoding tries over mixed messages and sending rehearses over mixed and encoded messages. The tight blend of encoding, encryption, and sending makes the purpose of constrainment structure ably meet the necessities of content control, information riddle, and information sending.

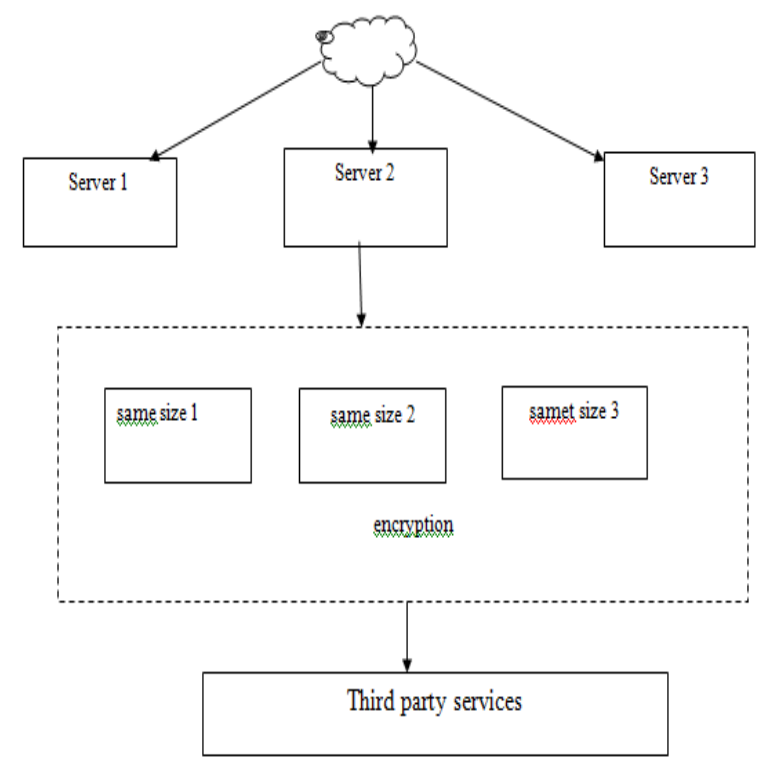

\section{SUMMARY}

In this section, we exhibited our estimations and perceptions from information group underway that stores several petabytes of content over several 1000 gadgets, focusing on the detachment experiences and the impact of using; annihilation, codes (explicitly, RS codes) on the framework, system of a server ranch. In our estimations, we watched a center of more than 50 machine detachment events, for every day. These detachment, estimations, confirm, the past reports from other server ranches, concerning unavailability, being the standard rather than the unique case.

The examination of the estimations in like manner revealed annihilation program data, one frustrations, in a long shot overpowering circumstance. Moved, concentrate, propelling entertainment disillusionment, going with parts.

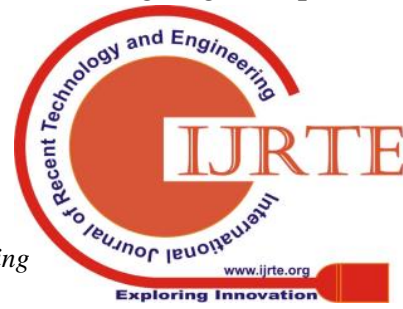


In like manner seen that the far reaching proportion of data in the midst of generation, squares eats up essentially measure framework content transfer limit and explicitly, puts additional load on the authoritatively, gives a genuine motivation to our work on amusement profitable, erasure codes for appropriated amassing, presented. . In the present system we oversees, data are moved in different servers with different, size. No tremendous security gave in the Cloud server to content prosperity. Basically one server utilized for verifying information. So single point disillusionment occurred. That is powerlessness to any data in a singular server influence the whole structure. Everything considered all data episode from the server. In the wake of using different servers this issue could be avoided The encryption concocts in the present structure are not feasible, in light of the fact that essentially couple of errands, are reinforced over mixed data. In case at all security exists, the pariah examiner, should be allowed to get to the entire, data packs for attestation. Cloud encounters, an interminable frustration and loses most of its data there is no inducing, assistance process in the present structure.

In the proposed framework, Cloud server will part the report into packs, and thought about encryption. The isolating, blended social gatherings are kept in various, Cloud servers and their keys, are passed, on in various key server. This blended content are changed over into bytes and included, regard bit process by the content owner to tie TPA by getting to the principal, information. The Cloud server, makes the token, number from the consistency included, encoded content and ascended out of the scratching, outfitted with the TPA, to check the Content Integrity. Additionally perceive, for the fortification, of the information.

\section{CONCLUSION}

In this task, issue of information security in cloud information stockpiling was explored, which is basically an appropriated stockpiling framework. To accomplish the affirmations of cloud information trustworthiness and accessibility and implement the nature of reliable distributed storage administration for clients, a successful and adaptable dispersed plan with unequivocal unique information support, including square refresh, erase, and add proposed. Deletion amending code in the record circulation arrangement to give repetition equality vectors and assurance the information constancy. Utilizing homomorphism circulated determination eradication lined value; Decision uses the concurrent ID of the making trouble server(s).

\section{FUTURE WORK}

we implement content are stored and uploaded into different servers they are encrypted content in same size. But in the future work we encrypt the content using different encryption in each servers they provide high security. Reliability of the system is very high.

\section{REFERENCES}

1. I. S. Reed and G. Solomon, "Polynomial codes over certain finite fields," J. Soc. Ind. Appl. Math., vol. 8, no. 2, pp. 300-304, 1960.
2. J. S. Plank and L. Xu, "Optimizing Cauchy ReedSolomon codes for faulttolerant network storage applications," in Proc. IEEE Int. Symp. Netw. Comp. Appl., 2006, pp. 173-180.

3. M. Blaum, J. Brady, J. Bruck, and J. Menon, "EVENODD: An efficient scheme for tolerating double disk failures in RAID architectures," IEEE Trans. Comput., vol. 44, no. 2, pp. 192-202, Feb. 1995.

4. P. Corbett, et al., "Row-diagonal parity for double disk failure correction," in Proc. 3rd USENIX Conf. File Storage Technol., 2004, pp. 1-14.

5. R.Durga and P.Sudhakar, "Recent Developments in Progress on Network Security Using Cryptography and Wireless Security" Paper is published in the journal of " Jour of Adv Research in Dynamical \& Control Systems, (SCOPUS) Elsevier" Vol. 9, No. 4, 2017.

6. Y. Fu and J. Shu, "D-Code: An efficient RAID-6 code to optimize I/O loads and read performance," in Proc. IEEE Int. Parallel Distrib. Process. Symp, 2015, pp. 603-612.

7. Y. Wang, X. Yin, and X. Wang, "MDR codes: A new class of RAID-6 codes with optimal rebuilding and encoding," IEEE J. Sel. Areas Commun., vol. 32, no. 5, pp. 1008-1018, May 2014.

8. J. S. Plank, "The RAID-6 Liberation codes," in Proc. 6th USENIX Conf. File Storage Technol., 2008, pp. 97-110.

9. R.Durga and P.Sudhakar, "Designing and Enhancing of network detection and security algorithm to implement the protocol with simulation of the optimal channels", IEEE international conference, Publish in IEEE Xplorer, INDIA Com - 2018, New Delhi.

10. M. Blaum and R. M. Roth, "On lowest-density MDS codes," IEEE Trans. Inf. Theory, vol. 45, no. 1, pp. 4659, Jan. 1997

11. Y. Zhu, J. Lin, P. P. C. Lee, and Y. Xu, "Boosting degraded reads in heterogeneous erasure-coded storage systems," IEEE Trans. Comput., vol. 64, no. 8, pp. 2145 2157, Aug. 2015.

12. C. Huang and L. Xu, "STAR: An efficient coding scheme for correcting triple storage node failures," IEEE Trans. Comput., vol. 57, no. 7, pp. 889-901, Jul. 2007.

13. R.Durga and P.Sudhakar, "Cryptographic Approach For Data Transfer Using Protocols" is published in the journal of "International Journal of Applied Engineering Research”, ISSN 0973-4562 Vol. 10 No.82 (2015) ( Research India Publications.

14. Henry C.H. Chen and Patrick P.C. Lee "Enabling Content Integrity Protection in Regenerating -CodingBased Cloud Storage: Theory and Implementation" Ieee transactions on parallel and distributed systems, VOL. 25, NO. 2, February 2014.

15. Almas Ansari, Prof. Chetan Bawankar, "Privacy \& content integrity for secure cloud storage," IOSR Journal of Computer Science (IOSR-JCE)

16. Giuseppe Ateniese "Remote Content Checking Using Provable Content Possession" ACM Transactions on Information and System Security, Vol. 14, No. 1, Article 12, Publication date: May 2011. 